\title{
Study on Measurement Method of Material Elastic Index
}

\author{
Yanxia Wang, Hai Yun*, Tiantian Sun, Qinghui Shang, Yongli Bao \\ School of Traffic and Vehicle Engineering, Shandong University of Technology, \\ 255049, ZiBo, china.
}

E-mail: wangyx200@163.com

\begin{abstract}
Keywords: Elastic index; steel; Mullite ceramic; ultrasound wave; measurement method; engineering mechanic experimental; engineering mechanic teaching.
\end{abstract}

\begin{abstract}
Elastic index is the required important parameters in the theoretical analysis and design calculation of components. Mechanical testing method and electrical measuring method are commonly used in engineering mechanics experimental. This article discusses the ultrasonic method which is used to measure the material elastic index. In this paper, Using ultrasound method to measure the elastic index of 1095 steel, and comparing with literature values, ultrasound measurement method and electrical measurement method are used to measure modulus of elasticity of Mullite ceramic. Experimental results show that the ultrasonic measured elasticity coefficient value is very similar with literature value; Comparing the two groups of experimental data,it is found that data obtained by ultrasound measurement method is compact while data obtained by electrical measurement method is dispersed and the average value of two groups variate within 5\%. This method has benefits such as no use of strain gauge,low costs, short cycle and multi-point measurement, thus it is suitable for multi-person experiment. In addition, this method can broaden students' knowledge horizon and cultivate independent thinking ability and practical ability.
\end{abstract}

\section{Introduction}

Elasticity indicators Including the young's modulus of elasticity, poisson's ratio, shear modulus of elasticity are the important need parameters in process of theoretical analysis and design calculation. In the present course of "engineering mechanics", the elastic index of the material is mainly determined by mechanical testing method or electrical measuring method[1,2]. But mechanical testing method is big costs due to use a specimen for measure once. Electrical measuring method requires students to accept training and test cycle is long, so the demand to the student ability is high.

In this paper, ultrasonic method was used to measure the material elastic index. The method by measuring the density of material, the material of transverse wave and longitudinal wave propagation velocity, is used to calculate the material elastic performance indicators such as shear modulus, young's modulus of elasticity and poisson's ratio, etc. This method broadens students' knowledge horizon and improves students' experiment analytical abilities, being suited for promotion in college engineering mechanical curriculum with fewer classes and more students participate in experiments.

\section{Experiment Method}

Experimental Theory. The calculation formula of poisson's ratio, shear modulus and elastic modulus, which is be derivated by the material constitutive equation, continuity equation and particle motion equation, can be expressed by wave velocity[3-5]:

$$
\left.\begin{array}{l}
\mu=\frac{1}{2} \frac{c_{L}^{2}-2 c_{T}^{2}}{c_{L}^{2}-c_{T}^{2}} \\
G=\rho c_{T}^{2} \\
E=\rho c_{T}^{2}\left[\frac{3 c_{L}^{2}-4 c_{T}^{2}}{c_{L}^{2}-c_{T}^{2}}\right]
\end{array}\right\}
$$


$C_{T}$ is velocity of transverse wave, CL is velocity of longitudinal wave, $\mathrm{E}$ is modulus of elasticity, $\rho$ is density of specimen.

It is shown in formula (1) that elastic parameters such as shear modulus, Young's modulus, and Poisson's ratio of materials can be calculated by the measured value of density, velocity of transverse and longitudinal wave. In addition, the properties of materials can be evaluated indirectly by measuring sonic velocity of materials because elastic coefficient is function of sonic velocity.

The operation of measuring ultrasound velocity of propagation according to standards ASTM C 1419[6,7] is shown in Fig(1). Ultrasonic velocity can be calculated by measuring height $h$ and ultrasonic propagation time $t$ of specimens having parallel surface. The formula of measuring velocity is as follow:

$$
v=\frac{2 h}{t}
$$

$2 h$ is distance of ultrasonic propagation path, $t$ is propagation time.

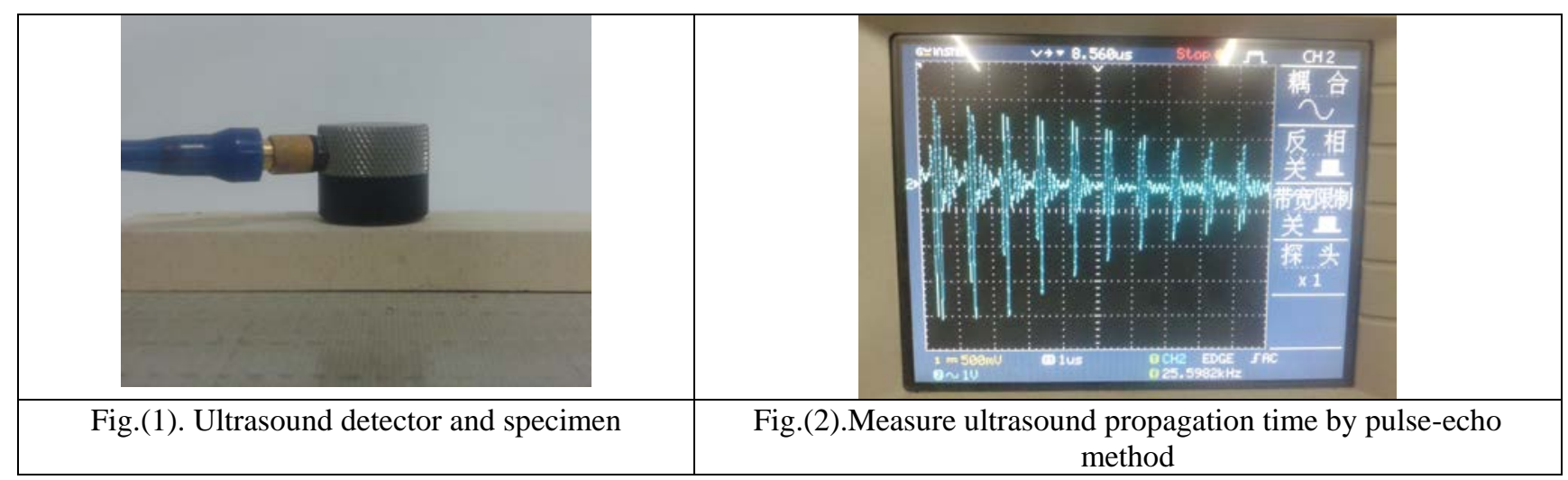

Measurement of sonic velocity is the key point of ultrasound measurement method[8-11]. Accuracy of experiments can be improved by measuring ultrasound propagation time in specimens repeatedly with pulse-echo method, which is shown in Fig (2). Pulse-echo method aims to pick two adjacent peak or trough value and then subtract them using reflection wave on interface. Because the shapes of specimen are regular, measurement of density can be carried out through dividing mass of specimen weighted by electrical balance by volume.

Instrument Operation. Instruments used in ultrasound test include OLMPUS pulse generator, preamplifier, numerical oscilloscope, shear wave probe,coupling agent, vernier caliper, electronic balance instrument.

Instruments used in electrical measurement method include universal testing machine,static strain gauge,digital electrical strain gauge.

\section{Experimental Data and its Analysis.}

Experimental Data. Values of elastic parameters of steel 1095 measured by ultrasonic method and obtained from literature respectively are shown in Table 1.

Table 1: elasticity parameters obtained from experiments and literature

\begin{tabular}{|c|c|c|c|}
\hline Steel 1095 & Num. & Num. & Lierrature value \\
& 1 & 2 & \\
\hline Elastic modulus E/GPa & 205.2 & 208.2 & 206.6 \\
\hline Poisson's ratio $\mu$ & 0.208 & 0.218 & 0.208 \\
\hline Shear modulus G/GPa & 79.22 & 79.87 & 79.57 \\
\hline
\end{tabular}

Elastic modulus of honeycomb ceramics measured by ultrasonic method and electrical method are shown in Table 2. 
Table 2: elasticity modulus of honeycomb ceramics

\begin{tabular}{|c|c|c|c|c|c|c|}
\hline Elstic modulus & Num. & Num. & Num. & Num. & Num. & Average value \\
& 1 & 2 & 3 & 4 & 5 & \\
\hline Ultrasonic method E/GPa & 26.85 & 26.74 & 26.92 & 26.65 & 26.78 & 26.79 \\
\hline Electrical method E/GPa & 27.70 & 28.31 & 28.35 & 27.37 & 29.07 & 28.16 \\
\hline
\end{tabular}

\section{Analysis of Experimental Data.}

(1) Comparing elastic modulus of steel 1095 measured by ultrasonic method with values obtained from literature, it is found that experimental values obtained by experiment are approximate to literature values thus ultrasonic method is suitable for measurement of elastic modulus. Ultrasonic method is recommended for an effective method of measuring elastic modulus of metal for its benefit such as simple experimental process and precise data.

(2)Comparing elastic modulus of honeycomb ceramics measured by ultrasonic method and electrical method, we find that values obtained from electrical method are discrete while that of ultrasonic method are concentric and both methods meet the needs for the average difference of the values obtained by two methods is under $5 \%$.

(3) Measuring elastic modulus of metallic and non-metallic materials by ultrasonic method will have more applications in fields of elastic modulus measurements for its advantages such as small error, simple operation,high accuracy.

(4)Viewing from values measured by ultrasonic method and electrical method, ultrasonic method is suitable for measurements of elastic parameters such as elastic modulus, Poisson's ratio, shear modulus and etc.

\section{Summary}

Restricted by the class hours the engineering mechanic experiments are mostly carried out by mechanical and electrical method. Mechanical method is suitable for mechanical property measurements in laboratory because the method is simple but requires huge devices, thus this method is usually used for specimens with simple structure. Electrical measurement has a long period and operation is relatively difficult for students, in addition new strain gauges are used in each measurement resulting the high costs of experiments. Stress distribution of complicated structures can be obtained by ultrasonic method and the measurement range is wide. Devices are convenient for carrying and this method can avoid students copying experimental data for measuring positions of each experiment are different. Ultrasonic method can broaden knowledge horizon and consolidate theoretical knowledge of the students, thus it is worthy of promotion.

\section{Acknowledgement}

This work was financially supported by the excellent course in Shandong Province "Engineering Mechanics", a grant from National High Technology Research and Development Program of China (2009AA063202) and the Shandong Province Natural Science Fund (ZR2009FQ023, ZR2011EL017, ZR2013EEQ008).

\section{References}

[1] Hongwen Liu, Material mechanic[M]. Beijing: higher education press, 2011.

[2] Dai Baoqing, Zhang Donghuan. Engineering mechanics[M]. Beijing: Beijing University of Aeronautics and Astronautics Press, 2010

[3] Zhilun Xu. Elasticity mechanic[M]. Beijing: Higher education press, 2010.

[4] Zuoyong He, Yufang Zhao. Basical theory of sonic[M]. Beijing: Defense industry press, 1981. 
[5] Jiankai Hu, Qianlin Zhang. Principals and methods of ultrasound test[M], Anhui: University of technology of China press, 1993.

[6] Fangcheng He, Zhaobin Gong. Ultrasound measurement technology and its application in material evaluation[J]. Material engineering, (8): 32 34.

[7] Yifang Chen, Jiajun Zhang. Research on ultrasound detection of modulus of elasticity[J]. Nondestructive detection, 1997, 19(9): 241 244 .

[8] Yiwei Shi. Ultrasound detection[M]. Beijing: Mechanical industry press. 2005.

[9] Paul M, Albert S B, Robert E G. Nondestructive Testing Handbook-Ultrasonic Testing[M], 1996: 224

[10] Yinlei Zhou, Mingshui Zhao, Qian Liang. Measurement of modulus of elasticity of roll by ultrasound method[J]. Nondestructive detection, 1994, 16 (12): 347 350 .

[11] Yongchang Chen. Ultrasonic nondestructive evaluation of thermal fatigue damage[D]. Dalian: Dalian university of technology press, 2007 